\title{
EL GÉNERO EN COMBATE: LAS ESTRATEGIAS DEL CUERPO EN LAS PRIMERAS PERFORMANCES DE VALIE EXPORT
}

\section{Carlos Tejo Veloso}

Universidade de Vigo, Dpto. Pintura

\section{Resumen}

Este artículo propone rescatar, reivindicar y analizar los primeros trabajos de la artista Valie Export (Linz, 1940) llevados a cabo durante las décadas de 1960 y 1970. Además de su indiscutible calidad artística y trascendencia política, la importancia de estas primeras performances de Export radica en la inteligencia y originalidad con que la creadora supo traducir al campo artístico los principios ideológicos de la lucha feminista del momento. Será precisamente en esta época, cuando brota con fuerza un arte producido por mujeres que denuncia con valentía los desmanes de una sociedad abiertamente machista. Dentro de este colectivo de artistas, la obra de Export mantiene una personalidad destacada, revelándose como una de las poéticas más inteligentes e influyentes de su tiempo.

Palabras clave: VALIE EXPORT (1940-); PERFORMANCE; ARTE FEMINISTA

\section{GENDER IN COMBAT: THE STRATEGIES OF THE BODY IN THE EARLY PERFORMANCES OF VALIE EXPORT}

\begin{abstract}
This article proposes to rescue, vindicate and analyse the early works of the artist Valie Export (Linz, 1940) carried out during the 1960s and 1970s. In addition to their unquestionable artistic quality and political significance, the importance of these first Export performances are based on the intelligence and originality with which the creator was able to integrate the ideological principles of the feminist struggle into the artistic field. It will be precisely at this time, when art produced by women springs forth forcefully to denounce with courage the excesses of an openly macho society. Within this collective of artists, the work of Export maintains an outstanding personality, revealing itself as one of the most intelligent and influential poetics of its time.
\end{abstract}

Keywords: VALIE EXPORT (1940-); PERFORMANCE ART; FEMINIST ART

\footnotetext{
Tejo Veloso, Carlos. 2019. "El género en combate: Las estrategias del cuerpo en las primeras performances de Valie Export“. AusArt 7 (2): 113-127. DOI: 10.1387/ausart.21153
}

\section{AUSART}




\section{INTRODUCCIÓN}

Permítasenos comenzar con un breve apunte que pueda aclarar el significado que le damos a la palabra performance dentro de la investigación en arte. Este término, que en España encuentra su hermano gemelo en la locución 'arte de acción', se utiliza internacionalmente para tratar de definir una práctica artística cuyos inicios se sitúan en las vanguardias históricas bajo la influencia del surrealismo, el dadaísmo, el teatro de vanguardia o la danza más rupturista. Con todo, si bien en esta época se fraguan algunas de las estrategias del género, no será hasta la década de 1950 cuando la performance comienza a tener cierta autonomía en el panorama artístico occidental (Glusberg 1979).

En relación a su semántica, es importante no caer en la confusión que a menudo produce el polivalente significado del término en lengua inglesa. Como sabemos, las acepciones más comunes de la palabra performance en ese idioma son: representación, actuación o espectáculo. Así, el mundo anglohablante la utiliza indistintamente para denominar cualquier actuación frente a un público (teatro, música, poesía, danza, etc). Contrariamente, el sentido de la palabra dentro del campo de la creación contemporánea es mucho más preciso y tomará prestado el término rechazando su polisemia. Siguiendo esta premisa, cuando hablemos de performance nos estaremos refiriendo a una práctica artística no objetual y multidisciplinar, de carácter efímero, que utiliza el cuerpo del artista o de una o más personas dirigidas por el artista, que se realiza frente a un público que podría llegar a ser participante, que tiene una duración variable y que puede llevarse a cabo en cualquier tipo de espacio: el campo, el espacio urbano, el interior de una galería o museo, etc (Martel 2009).

A partir de la segunda mitad del pasado siglo, la performance se realiza en entornos y países muy variados. Sólo por dar algunos ejemplos, recordemos el magnífico trabajo del colectivo japonés Gutai, el grupo Fluxus (con origen en EUA) o personalidades bien conocidas como el alemán Joseph Beuys o la artista de origen serbio Marina Abramovic.

En esta breve reflexión sobre la naturaleza de la performance no queremos pasar por alto el carácter comprometido y alternativo que presenta -o casi mejor sería decir, presentaba- la disciplina. El hecho de no producir obra objetual (pintura, escultura, fotografía o video), sitúa a la práctica fuera del mercado y de los circuitos oficiales que representan a la institución arte. Aunque esta situación periférica está poco a poco cambiando pues la performance está 
en un proceso imparable de institucionalización, nos interesa recordar que la significación política de la performance en el momento en que Export utilizaba esta manifestación artística era radicalmente diferente. En las décadas de los sesenta y setenta la performance se rebelaba contra el mercado y se movía por circuitos alternativos donde el feminismo tenía un protagonismo esencial (Phelan 2005). De signo indómito, también defendía un posicionamiento ideológico que no sólo iba en contra del mercantilismo artístico, sino que denunciaba las desigualdades de género, las dramáticas consecuencias que la guerra de Vietnam producía en la sociedad norteamericana o el derrumbe de la utopía moderna. La carga conceptual de la obra se sostenía sobre plataformas diversas como el dolor y la herida (Gina Pane, Chris Burden), la catarsis (Günter Brus), la transformación física (Orlan) o el rito (Ana Mendieta). El cuerpo y su presencialidad se constituía como un arma privilegiada en esta lucha; cuerpos abyectos que, cuestionando la norma, denunciaban las carencias de una sociedad en crisis.

\section{LO PERSONAL ES POLÍTICO: PERFORMANCE Y FEMINISMO}

Betty Friedan fue una de las primeras voces de Norteamérica en cuestionar que llevar a cabo las tareas domésticas tuviese como consecuencia un sentimiento gratificante o liberador. Para Friedan, los papeles de ama de casa y de esposa pasiva y sumisa podrían llegar a ser los principales responsables de un alto nivel de frustración, estrés y sometimiento; una extraña enfermedad que ella misma denominaría como: "The problem that has no name" $(1963,15)$. Esta idea, desarrollada en el libro The feminine mystique (1963), produjo un gran impacto en la sociedad americana de la época e influyó en el corpus ideológico del movimiento feminista conocido como la 'Second-wave feminism'. La trascendencia de esta obra clave es también notoria en el artículo de Carol Hanisch titulado "The personal is political" (1970). Hanisch combate aquí el planteamiento defendido por muchos hombres y mujeres americanas que, -aun reconociendo espacios de desigualdad -identificaban las protestas femeninas que aludían al sometimiento, alienación o discriminación con asuntos personales que nunca deberían de haber saltado a la arena política. Curiosamente, muchas de estas personas eran integrantes de un grupo progresista denominado Southern Conference Educational Fund (SCEF) con el que Hanish colaboraba. 
Años más tarde, la frase 'Lo personal es político' se convertirá en uno de los eslóganes de la "Second-wave feminism" y del "Radical Feminisim" (Puleo 2005). De forma sincrónica, el movimiento feminista en Europa gozaba de una larga tradición y fuerza en países como Reino Unido o Francia. A modo de ejemplo, pensemos en el Mouvement de Libération des Femmes (MLF.) francés o en la obra de intelectuales como la socióloga Colette Guillaumin (1995) o Simone de Beauvoir. Dentro del extenso legado de Beauvoir, su conocido libro El segundo sexo (1949) constituye una referencia ineludible. La situación general que rodeaba a estas mujeres de uno y otro lado del Atlántico se sustentaba, con lógicas diferencias, en una red común de desigualdades y dominación; entre todas estas aristas, nos gustaría destacar: la existencia de una pirámide de violencia contra la mujer que iba desde los micro-machismos al asesinato, la falta de leyes que garantizasen un aborto libre, gratuito y seguro, la exclusión de la mujer del mercado laboral y su consiguiente dependencia económica, una legislación del divorcio que privilegiaba al varón, un sistema educativo (sobre todo el superior) que vetaba el acceso de la mujer al conocimiento o la prácticamente inexistente participación de la mujer en cargos políticos relevantes.

Un grupo importante de artistas -muy activas en las décadas de 1960 y 1970 no quiso dar la espalda a estos problemas. Las creadoras se organizaron en pequeñas comunidades para poner en marcha colectivos de acción política, lugares de encuentro y debate o galerías alternativas. Estos nuevos espacios, alejados del carácter institucional de museos y galerías oficiales, llegaron a tener relevancia en ciudades como Nueva York, Los Ángeles, París, Londres o Berlín. En estos contextos, se utilizaba la performance para generar un arte nuevo y combativo que se oponía a un sistema del arte androcéntrico, anclado en un decadente formalismo (Cottingham 2000a,126). Llegamos así al germen de una idea que consideramos central: la tríada mujer-política-performance funcionará como una perfecta tribuna para visibilizar muchos de los problemas que denunciaba el feminismo norteamericano y europeo. Las creadoras luchan ahora por darle la vuelta al ambiente machista al que nos referíamos con anterioridad y recuperar así el control de su propio cuerpo. Como consecuencia, sus temáticas vindicaban el derecho al aborto y a los anticonceptivos, el derecho al divorcio y a la custodia de los hijos, el derecho a gozar de su sexualidad, el derecho a la igualdad en el trabajo o el derecho a sentirse seguras lejos de la violencia ejercida por el hombre. Dentro de la esfera del arte más activista, surgen colectivos y proyectos claves como las "Women's International Terrorist Conspiracy from Hell (W.I.T.C.H.)", "Womanhouse", o el "Woman's Builiding", entre otras experiencias. Mención especial merece el primer comisariado de arte feminista en Europa realizado por la propia Valie 
Export en el año 1975. El título de la exposición fue "MAGNA: Feminismus: Kunst und Kreativität". Entre las artistas más comprometidas del momento, no podemos olvidar el trabajo de Judy Chicago, Adrian Piper, Martha Rosler o Alexis Hunter. Conviene además recordar que, junto a esta lucha por los derechos fundamentales de la mujer, las artistas también se unieron para reclamar visibilidad en el establishment del arte y terminar así con esa injusticia histórica por la cual el arte y sus instituciones han privilegiado la imagen de un artista varón, todavía omnipresente en los libros y manuales de historia.

Esta orientación feminista en el arte se vio arropada por una pujante crítica de arte hecha por mujeres y preocupada por las mujeres. En esta corriente, debemos destacar nombres como Laura Cottingham (2000), Linda Nochlin (1971), Amelia Jones (2011) o Lucy Lippard (1980) entre muchas otras. El pensamiento feminista también comenzaba a encontrar un hueco en la enseñanza artística superior gracias a la aparición de estudios específicos. Especialmente relevantes fueron las experiencias llevadas a cabo por Judy Chicago y Miriam Schapiro. Chicago funda el primer programa de arte feminista en 1970 en Fresno, California. El año siguiente, junto a Miriam Schapiro, pone en marcha un programa similar en el California Institute of Arts. Estas actuaciones han ejercido una influencia cardinal en la posterior simbiosis entre arte y feminismo. Sus enseñanzas y sus reflexiones teóricas han formado a generaciones de artistas mujeres que, por primera vez, accedían a textos claves que despertarían su conciencia en pro de la igualdad de género. Tal y como apunta la profesora Peggy Phelam (2005), esta comunidad artística también se alimentaba de la ideología de los movimientos pacifistas y pro derechos civiles en los EUA, de las revueltas estudiantiles en Europa (especialmente del Mayo francés), de la creación en Europa y EUA de importantes colectivos feministas y de la influencia de pensadoras claves como las ya citadas, Friedan, Hanisch y Beauvoir, entre otras.

\section{DE HÖLLINGER A EXPORT: INFLUENCIAS, CONFLUENCIAS Y CONTEXTO}

En el año 1967, Valie Export decide modificar sus apellidos y sustituir el de su esposo por el nombre de una popular marca de cigarrillos en Austria. Waltraud Höllinger se convierte así en Valie Export. Este cambio, que nunca se pudo hacer oficial pero que -como ella misma señala en una entrevista- llegó a 
estar en uno de sus pasaportes (Kennedy 2016), comporta toda una declaración de principios. Con este gesto, Export arrinconará la oligarquía masculina de su estirpe eligiendo como nueva identidad un producto de amplio consumo masculino de dudoso beneficio para la salud; todo un inteligente guiño hacia el mercado institucional del arte y el patriarcado. Paralelamente al desarrollo de este acto performativo -todavía vigente hoy en día- VALIE anunciaba el comienzo de su actividad artística. Veamos ahora cómo era el entorno artístico y socio-político que la rodeaba en ese momento.

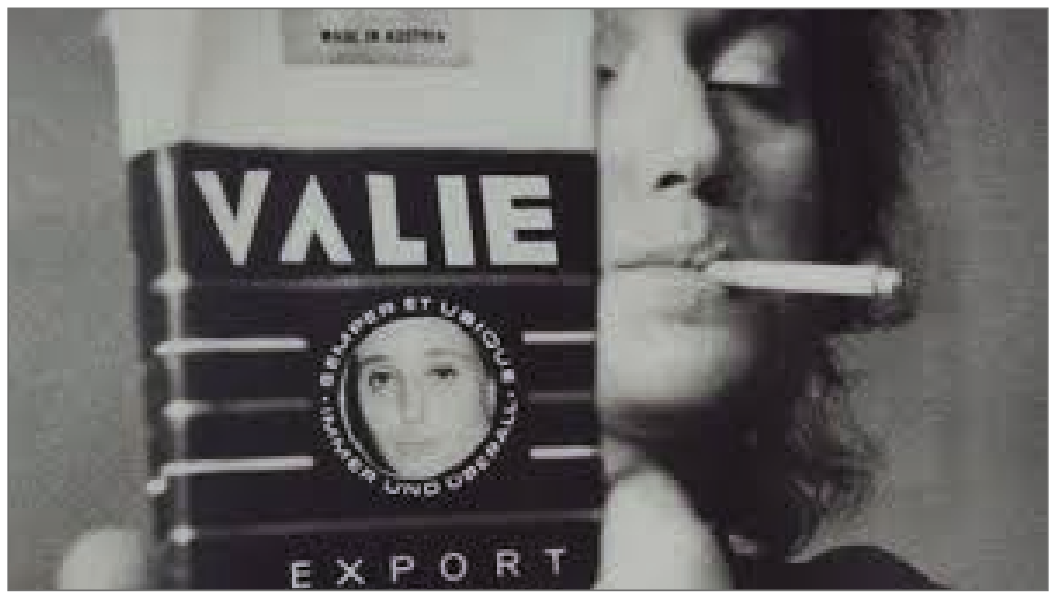

Fig. 1. Valie Export. Smart/Export, 1968, Impresión fotográfica

Entre la comunidad de artistas europeas y norteamericanas que trabajaban con planteamientos feministas en performance durante las décadas de los sesenta y setenta, había una relación importante que permitía un fértil intercambio de información y experiencias. En este sentido, Cottingham (2000a, 27) señala un intenso dialogo entre Export y Carolee Schneemann que, según la especialista norteamericana, no es más que un ejemplo de la simbiosis que tuvo lugar entre las mujeres que trabajaban arte y feminismo por aquel entonces. Del mismo modo, Wentrack (2014) nos confirma un fructífero encuentro entre las dos artistas en Londres, en el año 1970, cuando ambas presentaban diferentes performances en el Filmmaker's Cooperative Action. Otra muestra de reciprocidad entre Europa y América queda de manifiesto en un interesante texto de la propia Export (1989). En esta reflexión se hace evidente -no sólo la relación, sino la profunda admiración- de la creadora austriaca hacia grandes artistas feministas norteamericanas de su generación: la ya citada Schneemann, Alison Knowles, Charlotte Moorman o Joan Jonas. Reforzando esta teoría del contacto intercontinental, el académico Tilman Baumgärte (2018) afirma que la obra de Export bien pudiera inscribirse en un mismo grupo junto 
a otras artistas feministas coetáneas que usaban las mismas estrategias conceptuales y tenían los mismos objetivos a la hora de hacer arte. Baumgärte agrupa el trabajo de Export con el de Hannah Wilcke, Carolee Schneemann o Eleanor Antin. El profesor Aliaga (2007) también nos recuerda el profundo conocimiento de Export de la situación del feminismo en el mundo anglosajón y el manejo - por parte de la artista austriaca- de textos esenciales de Schneemann o Lucy Lippard. Estas similitudes y movimientos de ida y vuelta nos confirman que el arte feminista norteamericano llegó con fuerza a Europa y una vez aquí, supo crecer y evolucionar en los diferentes países donde echó raíces. Incluso en la blindada España del periodo final del franquismo, encontramos esta influencia del feminismo norteamericano en algunas de las performances desarrolladas por artistas como Fina Miralles, Olga Pijoan o Angels Ribé.

Es fácil deducir que la información circulaba y que las artistas de los sesenta y setenta compartían objetivos y principios ideológicos. Aunque consideramos que no es momento de entrar en detalle, sí que nos gustaría recordar algunos casos del arte feminista de la época que hayan podido influir en la trayectoria de Export. Hablamos de piezas emblemáticas como "Cut Piece" de Yoko ONO (del año 1964), "Meat Joy" de Carole Schneemann (del año 1964) o "Vagina Painting" de Shigeko Kubota (del año 1965). La década posterior también nos ha dejado obras cardinales que aproximan la creación de Valie con el contexto del momento. Entre muchas otras, recordemos "Red flag" de Judy Chicago (del año 1971), "Semiotics of the kitchen" de Martha Rosler (del año 1975), la serie "Catalysis" de Adrian Piper (de los años 1970-1971) o "Les tortures volontairies" de Annete Messager (del año 1972). A pesar de la visión un tanto esencialista del género planteada por algunas de estas artistas, reconocemos que su obra mantiene un fructífero dialogo con el trabajo de la artista austriaca. Todas ellas han dejado un legado cardinal para la evolución del arte feminista de las décadas de los ochenta y noventa. Sin embargo, las generaciones posteriores -más preocupadas por el discurso colonial, el post-estructuralismo o la herencia teórica de Buttler (1990)- plantearán una idea del género entendida como un constructo social donde factores como raza, situación económica u orientación sexual van a tener mucho que decir.

La Austria de los sesenta conformaba un contexto peculiar pese al fluido contacto entre creadoras y a la pujante corriente feminista que llegaba imparable

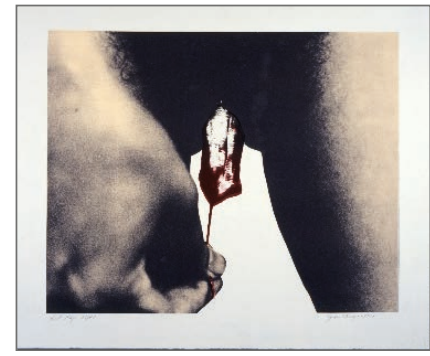

Fig. 2. Judy Chicago. Red Flag 1971, Impresión fotográfica 
desde Norteamérica. Después de la Segunda Guerra Mundial, se vivió una profunda crisis económica que afectaría a muchas esferas, incluidas el arte y la cultura. El país sufrió una dura postguerra caracterizada por la ocupación de su territorio por tropas de Francia, Inglaterra, la antigua Unión Soviética y EUA. Desde 1945 a 1955, Austria fue una región sin soberanía. En un intento de poner fin a esta situación, en 1955 el Parlamento del país aprueba la condición de ser un "Estado neutral a perpetuidad". Entre otras condiciones, este particular estatus le impide formar parte de cualquier alianza militar y le obliga a limitar su capacidad de respuesta bélica. Sin duda, esta neutralidad -que el profesor Rathkolb (2005) destaca como un efectivo código de internacionalización- avivó las contradicciones políticas de la sociedad de la época e influyó en la difícil construcción de una identidad nacional que pudiese olvidar, entre otras cosas, su pasado nazi. Al mismo tiempo, un tradicional catolicismo veía -no sin desagrado- como el aperturismo planteado por el Mayo francés y otros movimientos progresistas del momento se hacían hueco en el país.

En materia de cultura, tal y como apunta el investigador Guillermo Cano Rojas (2009), el arte austriaco de mitad de siglo pasado se situará en lo que algunos historiadores han denominado la "Hora Cero". En este periodo, se manejan dos posibilidades un tanto antagónicas: una vuelta nostálgica a la "Judendstill" y el rescate de figuras míticas del arte de vanguardia austríaco como Klimt, Schiele o Kokoschka o la asunción de una práctica artística más comprometida y actual que adoptase las estrategias propuestas por las corrientes internacionales del momento. Esta última opción -ligada a movimientos como Fluxus o a personalidades como Joseph Beuys- se alejará del formalismo que proponían movimientos como la "Abstracción Norteamericana" o el "Informalismo Europeo". Dentro de esta segunda vía, el provocador "Accionismo Vienés" tuvo un papel predominante. Al mismo tiempo, el trabajo de los "Accionistas" posibilitó que en la ciudad de Viena se crease un ambiente propicio para una arriesgada y novedosa experimentación artística donde la performance va a tener un papel fundamental.

El Accionismo Vienés fue un movimiento artístico que trabajó la performance durante la década de los sesenta, principalmente. Su principal objetivo abogaba por una apertura disciplinar que pudiese convertir al cuerpo y a la performance en signos de clara intencionalidad política opuestos a un formalismo adormecido. Muy críticos con la conservadora generación de postguerra austriaca, asumían las contradicciones políticas de su país utilizando sus performances como una suerte de terapia; como un proceso que les ayudase a superar las dramáticas consecuencias de la Segunda Guerra Mundial. En sus 
viscerales planteamientos creativos - muchos de ellos un marcado carácter misógino- atacaban las convenciones de la sociedad burguesa mediante la creación de ambientes donde el sexo, la sangre, la religión, el placer y el dolor, convivían estrechamente. Su trabajo mantuvo un carácter altamente subversivo, extremista, incluso en ocasiones, blasfemo. Nótese que, al hablar de este grupo, uno de los mayores errores es reducirlo a la obra desarrollada por los artistas varones Günter Brus, Otto Mühl, Hermann Nitsch y Rudolf Schwarzkogler. En este sentido, tal y como nos recuerda el profesor Sarmiento (2000), deberíamos entender este colectivo como una agrupación abierta a las colaboraciones entre sus miembros y heterogénea en sus planteamientos creativos. La participación de Export con el grupo encaja dentro de este parámetro más permeable; una participación tardía que prácticamente se redujo a una estrecha colaboración con Peter Weibel con quien llegó a desarrollar performances en el espacio urbano y también piezas en video como la conocida "Invisible adversaries" del año 1976. Valie Export, como señala en una entrevista con Rocío de la Villa (2004), nunca trabajó cercana al grupo accionista ni sintió que sus temáticas y su manera de enfocar la performance tuviese algo que ver con sus coetáneos. Desde un primer momento, manifestó su oposición hacia el machismo que destilaba el colectivo. Con todo, sí se vio especialmente influida por la atmósfera de aquellos años en la ciudad de Viena. A este respecto, la propia creadora, apunta (1982): "I was very influenced, not so much by Actionism itself, but by the whole movement in the city. It was a really great movement. We had big scandals, sometimes against the politique; it helped me to bring out my ideas".

\section{HACER PÚBLICO LO PRIVADO: LAS PRIMERAS PERFORMANCES DE VALIE EXPORT}

Export regaló una inteligente estrategia a las artistas feministas que le sucedieron: visibilizar lo privado en el espacio público. Este proceder sirve de hilo conductor para una obra de carácter multidisciplinar que, sobre todo durante sus primeros años, pone especial atención en la performance y en lo que ella misma ha denominado "Expanded Cinema"; un 'cine expandido' que privilegiará el contenido frente a la forma y que supondrá otra manera de acercase a la creación cinematográfica apoyándose en el video experimental, el uso no convencional de cámaras o la incorporación de materiales extra-cinematográficos. Dentro de este modo de hacer, Export ha producido obras importantes 
como la ya nombrada "Invisible adversaries" (del año 1976) o "Man \& Woman \& Animal" (del año 1973), entre otras.

"Aspects of feminist Actionism" es un importante texto de Export que conforma una significativa declaración de principios. En él se denuncia la situación subordinada de la mujer para, según la autora, dejar de ser: "the material 'woman', subjugated and enslaved by the male creator, into an independent actor and creator, subject of her own history" $(1989,71)$. Este pensamiento se convertirá en un concepto transversal en las performances que Export desarrolla a finales de los sesenta y durante la década de los setenta. Con un lenguaje directo, muchos de sus trabajos comprometen al espectador al abordar temáticas poco usuales para la época y, sobre todo, al situar al público frente a un sujeto femenino que rompe con los patrones de la burguesa y machista sociedad del momento. En este escenario, el espacio público presenta a la mujer como un agente activo de ruptura; una mujer que abandona el espacio doméstico y muestra su disconformidad con los roles de género impuestos por un esclerótico patriarcado. En referencia a esta idea, merece una mención especial la performance titulada "From the Portfolio of Doggedness" realizada en 1968. Como es habitual, este trabajo tiene una estructura simple y un mensaje directo. La performance se desarrolla por las céntricas calles de Viena y en ella podemos ver como Valie Export lleva de paseo -atado a una cadena de perro- a su más estrecho colaborador, Peter Weibel. Con grandes dosis de sentido del humor, la artista presenta un sujeto masculino en un estado de sumisión invirtiendo así los roles de género que normalmente se identifican con lo masculino; lanzando quizás una sarcástica llamada de atención al machismo que imperaba en los performers vieneses.

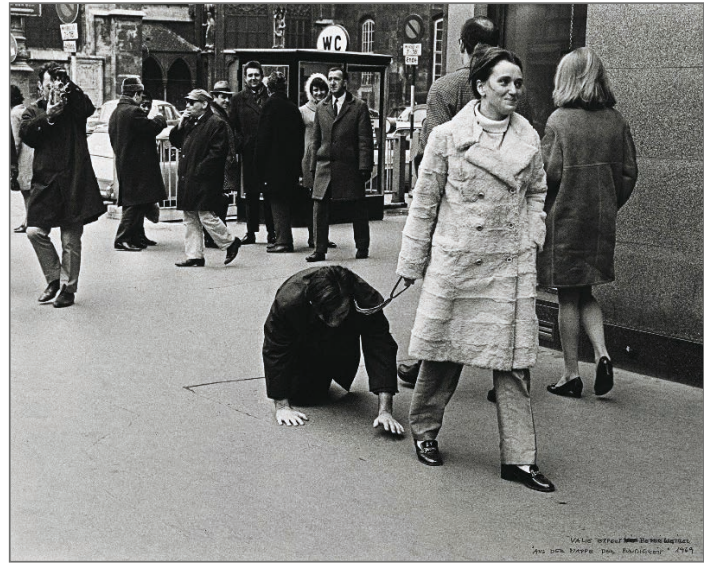

Fig. 3. Valie Export. From the Portfolio of Doggedness, 1968, Documentación fotográfica de la performance
Una sexualidad femenina consciente y orgullosa se convertirá en tema principal del arte feminista que se desarrolla en buena parte de Europa y Norteamérica durante los sesenta y setenta del siglo pasado (Wentrack 2014). En muchas ocasiones, estas prácticas utilizarán la imagen explícita del sexo femenino para -tras un ejercicio deconstructivo en la interpretación- reclamar otros significados que se opongan al 
morboso fetiche masculino (Jones 2011). Las artistas proponen otra manera de entender su sexualidad dinamitando los estrechos moldes marcados por la religión o el patriarcado; estamentos que reducen la actividad sexual en la mujer a un episodio pecaminoso, algo puramente reproductivo o a una fuente disciplinada de placer para el varón. Subvirtiendo estos dogmas, la performance "Tap and Touch Cinema", realizada por primera vez en 1968, toma el espacio público para denunciar cuestiones como el voyerismo masculino, la equiparación del cuerpo femenino con un objeto de consumo y entretenimiento o la violencia sexual contra las mujeres. La artista se sujeta al tronco una caja de unos $50 \mathrm{~cm} \times 70 \mathrm{~cm}$ con una abertura frontal a la altura de sus pechos. Esta abertura está cubierta por una especie de telón tras el cual se encuentran los senos desnudos de la artista. Valie Export, invita a los transeúntes a meter las manos y tocar sus pechos. La invitación era realizada, megáfono en mano, por Peter Weibel. Resulta especialmente interesante la interpretación que ofrece Roswhita Mueller (1994) cuando afirma que este trabajo parte de una clara alusión al sesgo machista en la industria del cine y pone en jaque a ese voyerismo del varón hacia un objetualizado cuerpo femenino. Bastantes años antes, la crítica feminista de cine Laura Mulvey (1975), ya señalaba que la dominación masculina convertía al cuerpo desnudo de la mujer en el cine en un objeto de deseo y de consumo; en una burda proyección del deseo masculino heterosexual. En "Tap and Touch Cinema", Export ataca el cómodo anonimato que produce la sala cinematográfica para hacer público un patológico deseo masculino hacia un sujeto femenino pasivo, sin voluntad ni poder de decisión. Abriendo un poco más la lectura de esta performance, advertimos que su emplazamiento en el espacio urbano juega con la posibilidad de que pudiesen participar también las mujeres. El ofrecimiento de los pechos de la artista hacia un público heterogéneo en su género podría desviar la lectura realizada por Mueller y permitirnos sospechar una sutil defensa de la diversidad sexual.

"Action Pants: Genital Panic," es el título de otra de sus performances más significativas. Realizada en 1969, esta acción discurre en un cine de arte y ensayo de Munich llamado "Augusta Lichtspiele". La artista entra en la sala ataviada con una camiseta, cazadora y unos pantalones a los que le ha cortado la tela a la altura del sexo. Camina por la sala haciendo coincidir sus desnudos genitales con las cabezas de unos desconcertados espectadores. Como en "Tap and Touch Cinema", Valie reniega de la mujer objeto y crea una zona de tensión que podríamos identificar con aspectos ya desarrollados por Mulvey (1975). El rol de la artista en esta performance resulta desafiante pero nunca llegará a ser tan agresivo como para imaginarnos a la creadora austriaca esgrimiendo una recortada. $Y$ ustedes se preguntarán, ¿Por qué saca- 
mos a colación el arma? La respuesta es sencilla. Alrededor de este trabajo, se ha creado una falsa mitología que sitúa a la artista en el interior de un cine porno apuntando al público con una ametralladora. Aunque reconocemos que esta versión le daría un giro interesante a la performance, esto no ha sido exactamente así. Autoras como Hilary Robinson (2013) o la ya referenciada Wentrack (2014) han contribuido a desmantelar esta falacia que sorprende por su popularidad (la hemos encontrado en no pocos artículos académicos y en páginas oficiales de museos como el MOMA o la Tate). Sospechamos que muchas de las críticas que relacionan esta acción con el "Accionismo Vienes" y que la cuestionan por reproducir un comportamiento demasiado agresivo y mimetizado con la violencia machista, están basadas en esta falsa creencia. Por otro lado, es probable que este malentendido se derive de las fotos realizadas por la propia artista un tiempo después de llevar a cabo la performance. En una de estas fotografías, Export aparece con los mismos pantalones, descalza, sentada en un banco con las piernas abiertas y sujetando una escopeta. De la performance no existe ninguna documentación conocida.

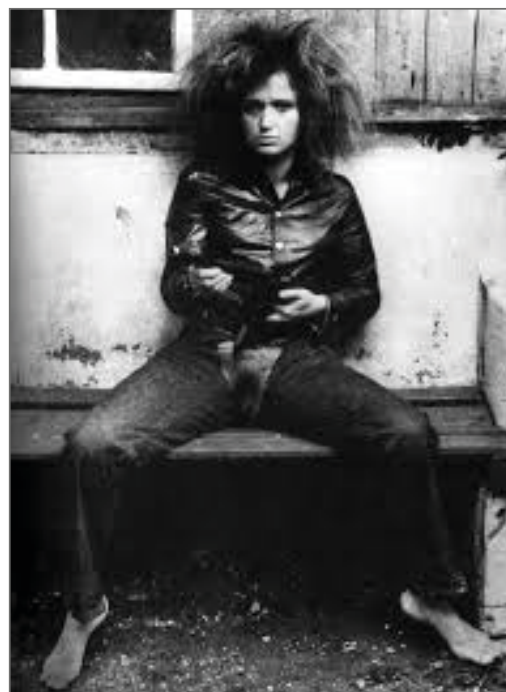

Fig. 4. Action Pants: Genital Panic. Valie Export, 1969, Impresión fotográfica
En su perfomance titulada "Eros/ion", realizada en el año 1971, Export sitúa el auto-castigo y el dolor en un primer plano de contenido, convirtiendo a su cuerpo en hacedor de una acción con una gran carga ritual y simbólica. La mecánica de la performance es sencilla: la artista desnuda se va colocando sobre tres superficies diferentes dispuestas horizontalmente sobre el suelo. La primera de ellas tiene una gran cantidad de pequeños cristales rotos, la segunda es un gran cristal sin romper y la tercera un trozo de papel. El cuerpo rueda consecutivamente sobre las tres superficies en un movimiento que se repite durante 10 minutos, aproximadamente. Las pequeñas heridas producidas por los cristales dejan un rastro de sangre que va transformando la apariencia del papel y del vidrio. Frente a otras performances de Export que utilizan más el absurdo o el sentido del humor, "Eros/ion" cambia de tercio y juega con los límites físicos para -acercándose a algunas performances de Abramovic- vehiculizar un mensaje que podemos relacionar con lo vulnerable, lo transitorio o lo cambiable. La violencia implícita en el acto de cortarse y la huella de la sangre son elementos que reducen la carga de erotismo a la que 
el cuerpo femenino está asociado. A nivel formal, resulta inevitable recordar las conocidas performances de Yves Klein en las que, cuerpos desnudos de mujer eran utilizados como pinceles para dibujar formas en azul sobre grandes lienzos en blanco. Subvirtiendo el superficial tratamiento del cuerpo femenino planteado por Klein, "Eros/ion" nos presenta una mujer que, activamente, utiliza el dolor como una metáfora del sometimiento. Derivadas y, en cierto modo, vinculadas a esta performance, Export realiza en 1973 tres trabajos especialmente significativos. Nos referimos a las performances tituladas: "Causalgia", "Hyperbulie" y "Asemia", descritas con detalle en el ya mencionado libro de Mueller (1994). Este grupo de acciones utilizan elementos comunes como cera, cable electrificado, juegos de luces y sombras, calor... Todas ellas coinciden en la necesidad de denunciar la opresión masculina y las limitaciones que la sociedad impone a la mujer en esa construcción machista de lo femenino. La idea del recorrido que describe la artista al desplazarse por diferentes estancias durante la performance, unifica esta triada y la relaciona con su trabajo anterior, "Eros/ion". La presencia de un texto en una de las partes de "Caulsagia" nos resume, con bastante precisión, el sentido de esta particular trilogía.

"Woman is forced to represent herself through jewellery, make-up, personality and as a bearer of fixed sexual symbols which are signs of a phallocratic society, in a way that does not correspond to her personal needs. Based on the system of biological differences, a sociological system of repression was erected, which woman can escape only by rejecting the body defined in this manner as feminine".

(Export, 1994, 36).

Junto a este conjunto de trabajos nos gustaría recordar otros títulos que presentan coincidencias conceptuales con los trabajos analizados como "Homometer" y "I am beaten", ambos de 1973 o "I [Beat (it)]" de 1978. Al lado de este cardinal corpus de performances, Valie Export nos ha dejado una trayectoria artística extensa e interdisciplinar. Nuestro análisis de las primeras performances de Export ha estado acotado a aquellos proyectos que encajan dentro de los parámetros de un arte efímero multidisciplinar que no produce obra objetual y que utiliza como herramientas: la semántica del cuerpo y el valor de su presencia, la incorporación del azar como estrategia creativa, el tiempo compartido con la audiencia, la influencia del contexto en el concepto, el espacio como continente y contenido y la posible participación del espectador como agente activo en cualquier momento del proceso. 


\section{CONCLUSIONES}

La irrupción de la performance feminista en EUA y en Europa en los años sesenta y setenta, significó una revolución política y artística que sacudió los cimientos de un arte burgués acomodado. Dentro de este contexto, las primeras performances de Valie Export marcaron un punto de inflexión muy importante en la lucha de la mujer artista por la igualdad y el respeto. Sus primeros trabajos, fuera del arte más mainstream y del apoyo de la crítica, participan de un periodo en el que una gran comunidad de mujeres artistas abraza los principios políticos del feminismo y decide poner su arte al servicio de la defensa de sus derechos y libertades. Advirtiendo la importancia de estos objetivos comunes, nos gustaría destacar que Valie Export se acercaba a la performance de una forma ingeniosamente diferente. En este sentido, las primeras performances de la artista austriaca derrochan frescura, humor y una inteligente ambigüedad que desmarca estas obras de un hacer, quizás más encorsetado, de sus coetáneas. Su lenguaje creativo evitará el esencialismo para trascender así categorías binarias; sorteando, de una manera muy lúcida, el manido cliché. Al lado de esta marcada personalidad, su obra apostó por un arte periférico, no comercial, arriesgado y fuera del circuito institucional; una valiente decisión que acabará cimentando la triada mujer-política-performance como un poderoso pilar del panorama del arte occidental. Valie Export crea una nueva corporeidad de lo femenino que consigue dinamitar la representación tradicional de la mujer haciendo posible el tránsito del cautiverio, al combate. Este tránsito será un signo fundamental del arte contemporáneo con orientación de género, de ahí su relevancia.

\section{Referencias bibliográficas}

Aliaga, Juan Vicente. 2007. "Caso de estudio: El laboratorio vienés". En Orden fálico: Androcentrismo y violencia de género en las prácticas artísticas del siglo XX, 213-41. Madrid: Akal

Baungärte, Tilman. 2018. "Schwarzers Postkarte". Taz Archiv (blog), 6 abril. http: //www.taz. de/!5511700/

Butler, Judith. (1990) 2001. El género en disputa. Ciudad de México: Paidós

Cano Rojas, Guillermo. 2009. "Las desilusiones corporales: Contribuciones de la sexualidad radical del accionismo vienés". Tesis Univ. Politécnica de Valencia

Cottingham, Laura. 2000a. "Are you experienced? Feminism, art and the body politic". En Seeing through the seventies: Essays on Feminism and Art, 117-31. London: Routledge

— . 2000b. "L.A. Womyn: The Feminist Art movement in Southern California, 1970-1979. En Seeing through the seventies: Essays on Feminism and Art, 161-74. London: Routledge 
De Beauvoir, Simone. (1949) 2017. El segundo sexo. Traducción de Alicia Martorell. Madrid: Cátedra

Friedan, Betty. 1963. The feminine mystique. Oxford: Norton \& Co

Glusberg, Jorge. 1979. The art of performance. New York: New York University

Guillaumin, Colette. 1995. Racism, sexism, power and ideology. London: Routledge

Hanisch, Carol. 1970. The personal is political: Notes from the second year; Women's liberation; Major writings of the radical feminists. New York: Radical Feminism

Jones, Amelia. 2011. "Generando problemas: Las artistas feministas ponen en escena el sexo femenino". Youkali 11: 46-54

Kennedy, Randy. 2016. "Who is Valie Export? Just look, and please touch". The New York Times, 6 abril. https: //www.nytimes.com/2016/06/30/arts/design/who-is-valie-export-justlook-and-please-touch.html

Lippard, Lucy. 1980. "Sweeping exchanges: The contribution of feminism to the art of the 1970s". Art Journal 40(1-2): 362-5

Martel, Richard, Bartolomé Ferrando, Joan Casellas \& Nieves Correa Rodríguez. 2009. "Performance: Arte vivo y en directo". Exit Express 47: 17-25

Mueller, Roswhita. 1994. Valie Export: Fragments of the imagination. Bloomington IN: Indiana University

Mulvey, Laura. (1975) 1989. "Visual pleasure and narrative cinema”. En Visual and other pleasures, 14-26. London: Palgrave Macmillan

Phelam, Peggy. 2005. "Estudio". En Arte y feminismo, ed. de Helena Reckitt; traducido del inglés por Gemma Deza Gil, 15-63. Nueva York: Phaidon

Puleo, Alicia. 2005. "Lo personal es político: El surgimiento del feminismo radical”. En Teoría feminista: De la llustración a la globalización; De la llustración al segundo sexo, Celia Amorós \& Ana de Miguel, eds., 35-67. Madrid: Minerva

Rathkolb, Oliver. 2010. The paradoxical republic: Austria 1945-2005. New York: Berghahn

Robison, Hilary. 2013. "Actionmyth, historypanic: The entry of Valie Export's aktionhose; Genitalpanik into art history". Paradoxa International Feminist Art Journal 23: 84-9

Sarmiento, José Antonio. 2000. El arte de acción. Las Palmas de Gran Canaria: Centro de Arte La Regenta

Valie Export. 1973. "Woman's Art manifesto". Neues Forum 228: 47

— . 1982. "Valie Export by Gary Indiana". Bomb Magazine 3. https: //bombmagazine.org/ articles/valie-export/

- . 1989. "Aspects of feminist actionism". New German Critique 47: 69-92

— . 2004. "Arte, estética y más: Entrevista a Valie Export", por Rocío de la Villa. Exit Express 2. http: //rociodelavilla.blogspot.com/2009/04/entrevista-valie-export.html

Wentrack, Kathleen. 2014. "Female sexuality in performance and film: Erotic, political, controIlable? The contested female body in the work of Carolee Schneemann and Valie Export". Konsthistorik Tidsskrift 83(2): 148-67 